\title{
Innovación docente en los seminarios de enfermería de la infancia y adolescencia
}

\section{Teaching innovation in childhood and adolescent nursing seminars}

Ma deL SOCORRo ARnEDILLO SÁNCHEZ

ORCID: https://orcid.org/0000-0002-7596-0749

Departamento de Enfermería

Universidad de Sevilla

marnedillo@us.es

Fecha de recepción: 19/11/2019

Fecha de aceptación: 21/11/2019

DOI: http://dx.doi.org/10.12795/9788447221912.089 Pp.: 2007-2028 
Se presenta un ciclo de mejora (CM) realizado en los seminarios de la asignatura "Enfermería de la Infancia y Adolescencia" del 2o curso del Grado de Enfermería. Se diseñaron mapas de contenidos y un modelo metodológico basado en el principio de investigación. Partiendo de la exploración de ideas de los alumnos se les muestran cuestiones desencadenantes y/o exposiciones de información. Estas se trabajan conjuntamente con el docente exponiendo las nuevas ideas de los estudiantes. Se resuelven casos clínicos de manera colaborativa. La resolución del caso incorpora como innovación la parte procedimental que clásicamente se realizaba en seminarios independientes, integrando de esta manera teoría y práctica. El docente concluye y recapitula las ideas principales. Se evaluó el CM identificando áreas de mejora y la adquisición de conceptos de alumnos mediante "escaleras de aprendizaje". La satisfacción de los alumnos fue muy alta.

Palabras Claves: Enfermería de la Infancia y Adolescencia, Grado de Enfermería, Docencia Universitaria, Experimentación Docente Universitaria, Seminarios.

\section{Abstract}

A teaching improvement cycle (IC) was carried out in the seminars of "Childhood and Adolescent Nursing", a subject taught in 2nd year of the Nursing Degree. Content maps and a methodological model, based on the research principle were designed. It started from exploring students' ideas, followed by presentation of trigger issues on the subject and / or information exposures. Student's new ideas were exposed after working the issues with the teacher. Clinical cases were solved collaboratively. The resolution of the case incorporated as an innovation the procedural part, which was classically carried out in independent seminars, thus integrating theory and practice. The teacher concluded and recapitulated the main ideas. The IC was evaluated and areas of improvement were identified. Assessment of student's concepts acquisition was carried out through "learning ladders". Student satisfaction with the seminars was very high.

Key words: Childhood and Adolescent Nursing, Nursing Degree, University Teaching, University Teaching Experience, Seminars. 


\section{Descripción del contexto}

La asignatura en la que se realiza el CM es "Enfermería de la Infancia y Adolescencia". Se trata de una asignatura obligatoria de segundo curso del grado de Enfermería y se imparte en el primer cuatrimestre. El CM se efectúa en los seminarios, cuya asistencia es obligatoria y tienen asignados tres créditos. El alumnado aún no ha tenido ningún contacto con entornos clínicos. Los seminarios están diseñados para abordar en forma de taller algunos aspectos de la teoría, con un componente procedimental importante. La cronología entre los contenidos teóricos y los seminarios no siempre es la adecuada, ya que hay aspectos que se abordan en los seminarios sin haber sido tratados en la teoría. Algunos contenidos son solo abordados en los seminarios.

El grupo en el que se realiza el CM está compuesto por 11 estudiantes, todas son mujeres. Los seminarios se imparten en el aula de simulación de la Facultad de Enfermería, campus de Perdigones. El aula es adecuada para el desarrollo de los seminarios ya que contamos con ordenador, proyector y todo el material necesario para llevar a cabo los procedimientos: maniquíes de simulación y material clínico de todo tipo. Trabajamos sentados alrededor de mesas grandes y altas que favorecen la interacción y el trabajo colaborativo.

\section{Diseño previo del ciclo de mejora}

\section{¿Para qué enseñar?}

Las unidades en las que se implementa el CM son: Vacunación en la Infancia y Salud Infantil- Cartilla de Salud Infantil. Girando en torno a dos cuestiones fundamentales. ¿Cuál es la función de la enfermera ante la vacunación?, ¿Qué medidas de cribado o profilaxis se realizan a todos los recién nacidos? Los objetivos de aprendizaje son: 
- Unidad 1 Vacunas:

- Describir las vacunas de aplicación sistemática en la Infancia y explicar las características de cada una de ellas.

- Explicar la actuación de enfermería antes, durante y tras la administración de la vacuna.

- Exponer las contraindicaciones absolutas, precauciones, falsas contraindicaciones y reacciones adversas de las vacunas.

- Practicar en el aula de simulación el procedimiento de vacunación.

- Valorar la importancia del consejo vacunal

- Unidad 2. Salud Infantil. Cartilla de Salud Infantil.

- Conocer Las medidas de cribado y profilácticas incluidas en la Cartilla de Salud Infantil.

- Explicar qué métodos existen actualmente para evaluar la audición en los recién nacidos.

- Describir las pruebas de cribado endocrino metabólicas. Actuación de enfermería.

- Determinar cuál es el tratamiento adecuado para la profilaxis ocular y de la enfermedad hemorrágica del recién nacido y cuándo se realiza. Actuación de la enfermera.

- Practicar en el aula de simulación las distintas técnicas.

- Valorar la importancia de las medidas profilácticas y de cribado en el recién nacido.

\section{¿Qué enseñar?}

Para tener presente a la hora de diseñar el CM los contenidos, se elaboran tablas de contenidos para cada una de las unidades. Estas tablas facilitan el diseño de las actividades y ayudan a separar los tres grupos básicos de contenidos a trabajar: conceptuales, procedimentales y actitudinales (Porlan, 2017). Independientemente de que a posteriori se trabajen de manera integrada. 


\section{Mapas de contenidos}

Con idea de ayudar a expresar de manera gráfica y explícita el conocimiento sobre las dos unidades se realiza un mapa de contenidos para cada una de ellas. En los mismos se visualizan los contenidos y las interacciones entre los mismos, destacando los contenidos principales y diferenciando entre los tipos de contenidos. Las figuras 1 y 2 presentan los mapas de contenidos de ambas unidades del CM, la leyenda es la misma para ambas.

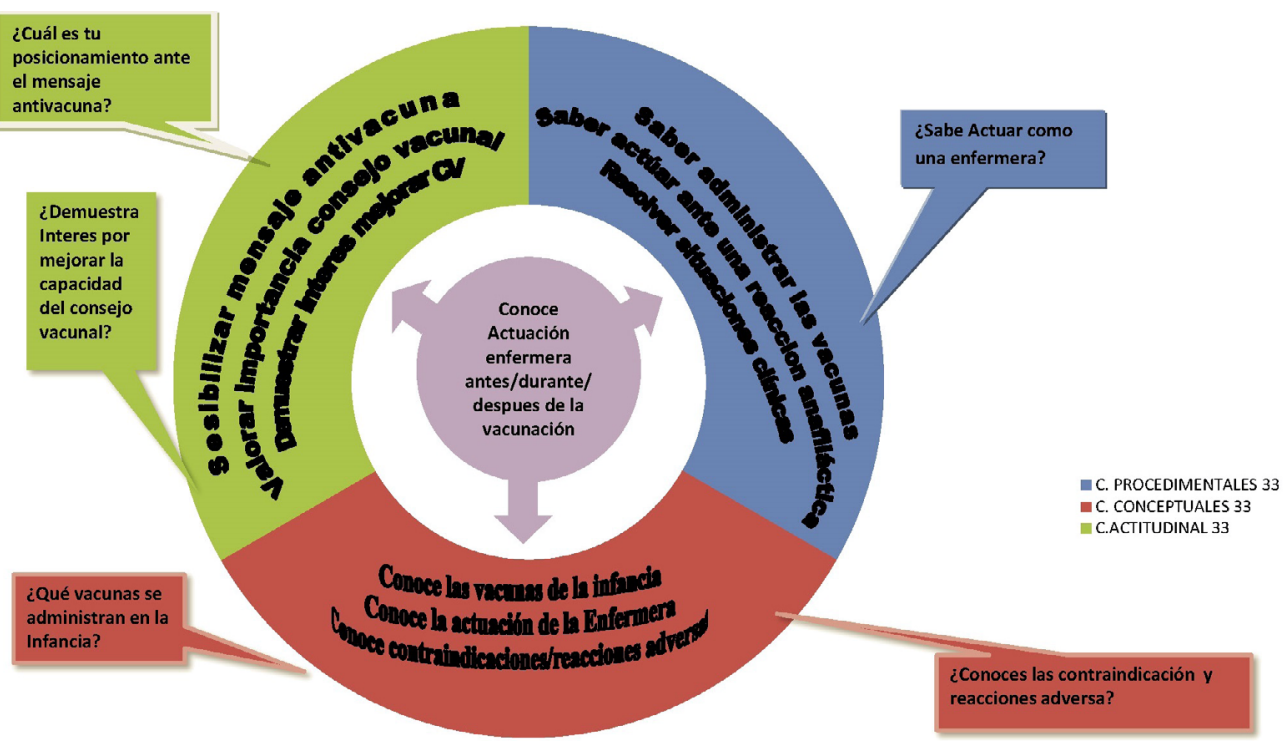

Figura 1. Mapa de contenidos Vacunas en la Infancia

Jornadas de Formación e Innovación Docente del Profesorado | № 2 (2019) Esta obra se distribuye con la licencia Creative Commons Reconocimiento-NoComercial-SinObraDerivada 


\section{Mą DEL SOCORRO ARNEDILLO SÁNCHEZ}

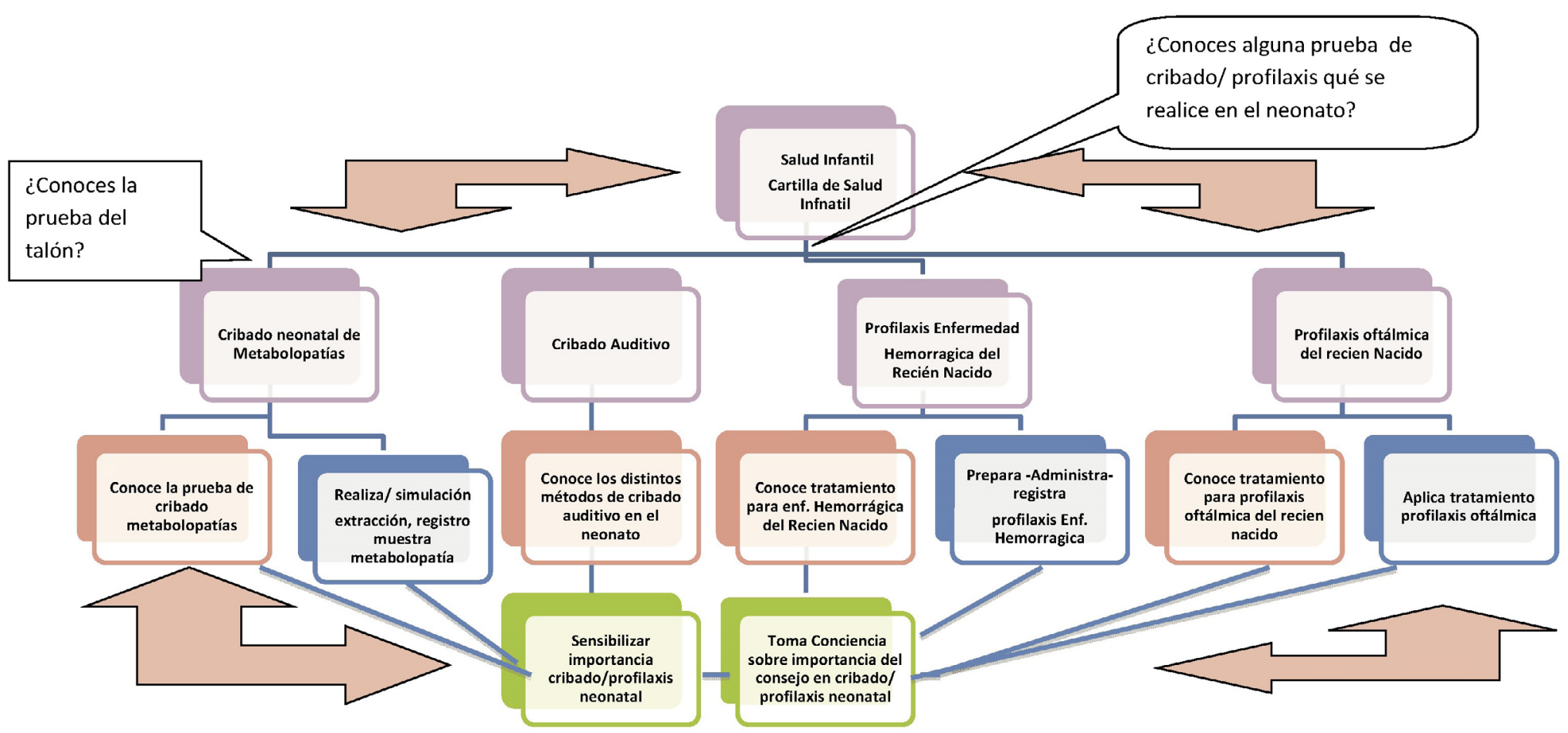

Figura 2. Mapa de contenidos Salud Infantil

Jornadas de Formación e Innovación Docente del Profesorado | № 2 (2019) 


\section{El modelo metodológico}

El modelo metodológico planteado se basa en el modelo de "aprendizaje por investigación" Proponiendo a los alumnos una cuestión que les suponga un reto y los active mentalmente, fundamental para la interacción y el intercambio de nuevas informaciones y elaborar las mejores respuestas a los problemas planteados (Porlan, 2017). La figura 3 presenta el modelo metodológico diseñado para el CM.

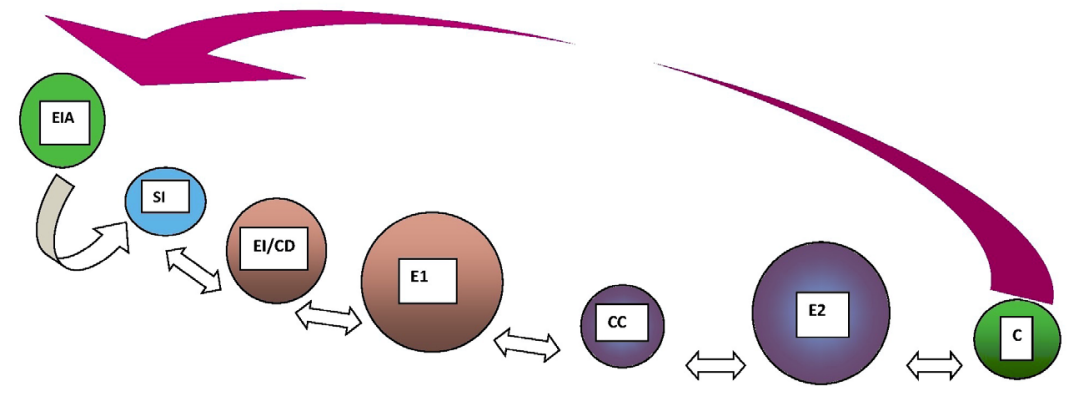

Figura 3. Modelo Metodológico

Así, en el modelo metodológico planteado se parte de la exploración de las ideas previas de los alumnos (EIA). Se empieza el ciclo con una sintesis de la sesión anterior e introducción al tema a tratar (SI). Se les presentan cuestiones desencadenantes (Finkel, 2018) y/o exposiciones de información (EI/CD) Estas se trabajan conjuntamente con el docente y se exponen las nuevas ideas de los estudiantes (E1). Con idea de reforzar y ampliar los contenidos planteados se presentan casos clínicos (CC) de interés (Bain, 2007) que los alumnos resuelven de manera colaborativa con la guía y apoyo del docente. A posteriori presentan la resolución del caso clínico que incorpora como innovación la parte procedimental, que clásicamente se realizaba en seminarios independientes, integrando de esta manera teoría y práctica (E2). El docente concluye (C) y recapitula las ideas principales. 
A continuación se presenta en la Tabla 1 la secuencia de actividades planteadas para la unidad de vacunas en la Infancia, coherente con al modelo metodológico planteado. Describiendo de manera detallada lo que va a acontecer en el aula (Porlan, 2017)

Tabla 1. Secuencia de las actividades unidad Vacunas en la Infancia

\begin{tabular}{|c|c|c|}
\hline \multicolumn{3}{|c|}{ TEMÁTICA 1 VACUNAS EN LA INFANCIA } \\
\hline Sesión & $\begin{array}{l}\text { Fase del Modelo } \\
\text { Metodológico }\end{array}$ & Actividades \\
\hline $\begin{array}{l}\text { Sesión Previa } \\
\text { CM }\end{array}$ & $\begin{array}{l}\text { ElA. Exploración de } \\
\text { las ideas previas } \\
\text { de los alumnos }\end{array}$ & $\begin{array}{l}\text { En una sesión previa al inicio del Ciclo } \\
\text { de mejora se pasa un cuestionario de } \\
5 \text { preguntas cortas sobre los temas a } \\
\text { tratar en el ciclo de mejora. }\end{array}$ \\
\hline Trabajo en casa & $\begin{array}{l}\text { El/CD. } \\
\text { Exposición de } \\
\text { Información. } \\
\text { Cuestión } \\
\text { desencadenante }\end{array}$ & $\begin{array}{l}\text { - Visualización en casa del documental } \\
\text { "Amor, Miedo y Vacunas". https:// www. } \\
\text { youtube.com/watch?v=0tdm3u1Yie8 }\end{array}$ \\
\hline \multirow[b]{3}{*}{1} & $\begin{array}{l}\text { IS Introducción } \\
\text { y sintesis sesión } \\
\text { anterior }\end{array}$ & $\begin{array}{l}\text { Sintesis de la sesión anterior } \\
\text { Presentación de la sesión }\end{array}$ \\
\hline & $\begin{array}{l}\text { E1. Exposición de } \\
\text { ideas }\end{array}$ & $\begin{array}{l}\text { - Respuestas individuales "Mentimeter" } \\
\text { video } \\
\text { - Análisis de los resultados en común. } \\
\text { - Exposición dialogada del docente } \\
\text { - Puesta en común. }\end{array}$ \\
\hline & $\begin{array}{l}\text { CC. Caso clínico de } \\
\text { interés }\end{array}$ & $\begin{array}{l}\text { - Se presenta al alumno con un caso } \\
\text { clínico relacionado con la vacuna de la } \\
\text { "Meningitis" } \\
\text { - Se lee el caso clínico por parte del } \\
\text { profesor. } \\
\text { - Se dan instrucciones precisas orales } \\
\text { y por escrito sobre los contenidos de la } \\
\text { resolución. } \\
\text { - Se presenta bibliografia para } \\
\text { resolución de caso. }\end{array}$ \\
\hline
\end{tabular}

Jornadas de Formación e Innovación Docente del Profesorado | № 2 (2019) Esta obra se distribuye con la licencia Creative Commons Reconocimiento-NoComercial-SinObraDerivada Internacional (CC BY-NC-ND 4.0.) 


\begin{tabular}{|c|c|c|}
\hline & $\begin{array}{l}\text { E2. Resolución del } \\
\text { caso aplicación } \\
\text { práctica de los } \\
\text { contenidos }\end{array}$ & $\begin{array}{l}\text {-Trabajo colaborativo de resolución del } \\
\text { caso clínico, grupos de } 4-3 \text { alumnos. } \\
\text { - Se expone la resolución del caso } \\
\text { clínico } \\
\text { - Un grupo realiza un rol-play de } \\
\text { la situación clínica. Incluyendo el } \\
\text { procedimiento. } \\
\text { - El docente comentará aportaciones } \\
\text { de alumnos, resaltando puntos críticos } \\
\text { de aprendizaje. }\end{array}$ \\
\hline \multirow{3}{*}{2} & $\begin{array}{l}\text { Cc. Caso clínico de } \\
\text { interés }\end{array}$ & $\begin{array}{l}\text { - Se presenta al alumno con un caso } \\
\text { clínico relacionado con las vacunas } \\
\text { para los distintos tipos de hepatitis. } \\
\text { - Igual a CC anterior }\end{array}$ \\
\hline & $\begin{array}{l}\text { E3. Resolución del } \\
\text { caso aplicación } \\
\text { práctica de los } \\
\text { contenidos. }\end{array}$ & $\begin{array}{l}\text { - Igual resolución anterior } \\
\text { - Análisis de Catillas de Vacunación de } \\
\text { los alumnos. Se comparan similitudes y } \\
\text { diferencias entre sí y con el calendario } \\
\text { actual. Toman conciencia de su estado } \\
\text { vacunal. Concepto de calendario como } \\
\text { algo dinámico según la epidemiología. }\end{array}$ \\
\hline & $\begin{array}{l}\text { C. Conclusiones y } \\
\text { recapitulación }\end{array}$ & $\begin{array}{l}\text { El docente concluye con las } \\
\text { aportaciones de los alumnos. }\end{array}$ \\
\hline
\end{tabular}

\section{Estudio de la evolución de las ideas de los alumnos}

Para analizar la evolución de los estudiantes, diseño un cuestionario de preguntas cortas básicas, que los estudiantes responderán antes de comenzar el ciclo y al final del mismo. Así mismo se realiza un juego de gamificación con la plataforma Kahoot de preguntas relacionadas con la unidad de las vacunas.

- Cuestionario de ideas previas de los alumnos:

- ¿Qué contraindicación permanente conoces para vacunar a un niño?

- Enumera todas las vacunas que conozcas que estén incluidas en el calendario de vacunación infantil.

- ¿Qué piensas sobre el debate actual en torno a las vacunas en la infancia?, ¿Sabes cuál es? 
- ¿Sabes en qué consiste la prueba del talón?, ¿La conoces por algún otro nombre, cuál?

- ¿Conoces alguna prueba de cribado o tratamiento profiláctica que se realice a todos los recién nacidos? Di cuales.

El análisis de las respuestas dadas en el momento inicial me hace detectar algunas cuestiones que me hacen replantearme y/o focalizar sobre ciertos contenidos.

Con respecto a las vacunas:

- Desconocimiento de muchas de las vacunas incluidas en el calendario de vacunación infantil

- Confusión sobre vacunas de enfermedades ya erradicadas - Viruela

- Concepto erróneo sobre vacunas disponibles para profilaxis de la meningitis y vacunación para los distintos tipos de hepatitis A, B y C.

Con respecto a la segunda unidad de salud infantil se aprecia un desconocimiento casi total de las medidas de cribado y tratamientos profilácticos en los recién nacidos. Los alumnos parten de un nivel más bajo al esperado. Decido por lo tanto adaptar el contenido a los niveles de conocimiento de los alumnos (Porlan, 2017).

Modifico los casos clínicos preparados, creando dos relacionados con los conceptos erróneos: vacuna "meningitis" y vacunas para la "hepatitis". El video que se utiliza en el seminario aborda el tema de la erradicación de enfermedades, con lo cual considero será suficiente para aclarar esta confusión. Decido hacer algún cambio en la exposición de contenidos para reforzar el aprendizaje en la unidad de Salud Infantil.

Jornadas de Formación e Innovación Docente del Profesorado | № 2 (2019) Esta obra se distribuye con la licencia Creative Commons 
Aplicación del Ciclo de Mejora

\section{Relato del desarrollo}

\section{Primer día}

Empezamos la sesión con una síntesis de la sesión anterior e introducción a la nueva. Hacemos un juego de gamificación con la plataforma Kahoot sobre algunos conceptos de vacunación. El uso de Kahoot siempre les resulta divertido y estimulante.

La primera actividad está relacionada con el video "Amor, miedo y vacuna". Es un documental sobre el movimiento antivacuna y expone de una manera científica y rigurosa los argumentos a favor de la vacunación en la infancia. Aborda aspectos sobre: evolución histórica, ventajas de las vacunas para el individuo, la comunidad y desmonta con evidencias los mitos sobre las vacunas. El 60\% de las alumnas ha visualizado el video en casa. Invito a las alumnas que no lo han visualizado a participar y verlo a posteriori.

Mediante el uso de la aplicación "mentimeter" analizamos los aspectos principales del video, su nivel de sensibilización hacia la importancia del consejo vacunal y los argumentos a favor del mismo. Es una dinámica que les gusta, no conocían la aplicación, pero se ven cómodas con el ejercicio. Exponen sus ideas, argumentan a favor de la vacunación usando los razonamientos empleados por la comunidad científica en el vídeo y se posicionan fuertemente a favor de la vacunación infantil. Resumimos los puntos fundamentales y pasamos a la siguiente actividad.

Leo a las alumnas un caso clínico relacionado con la vacunación -aborda la vacunación para la "meningitis", como han definido ellos en sus cuestionarios iniciales-. Doy instrucciones precisas orales y por escrito sobre la actividad que tienen que realizar y qué se espera de ellas al resolver el caso clínico. Muestro la bibliografia para la resolución del caso. 
Las alumnas se reparten en tres grupos de trabajo de 3-4 alumnos. Trabajan de manera colaborativa. Paso entre los grupos, me siento con cada uno de ellos para ver que están haciendo. Me preguntan sobre todo aspectos relacionadas con la simulación. Les informo que deben actuar como un profesional en consulta, llevando a cabo todos los pasos de la actuación de la enfermera.

Pasamos a la resolución de los casos. Contrastamos la información de cada grupo, y entre todas completamos una respuesta común y completa. Con respecto al rol play, al principio parecen tímidas, pero enseguida se meten en su papel. Preparan la vacuna y simulan los procedimientos. Es un momento divertido que provoca risas y comentarios sobre lo que va ocurriendo. El grupo hace observaciones sobre lo que ha pasado y las protagonistas también reflexionan sobre su actuación, a modo de debriefing. Revisamos todo el procedimiento, haciendo aportaciones sobre si falta algún paso, si las respuestas han sido adecuadas y cuál ha sido la actitud de las participantes según su rol.

Concluyo y resumo los pasos más importantes a tener en cuenta. Finaliza la sesión.

\section{Segundo día.}

Repetimos el ciclo anterior con la presentación de otro caso clínico. Esta vez relacionado con las vacunas existentes para las distintas formas de Hepatitis.

Los alumnos trabajan en los mismos grupos, esta vez se les ve más decididas, saben exactamente en qué tienen que hacer hincapié. Ya conocen la dinámica y la bibliografía y empiezan a resolver los casos con mucha soltura. Me siento con cada grupo para ver cómo trabajan y asesorar si me lo piden. Tras el tiempo de trabajo estipulado pasamos a la resolución de los casos. En esta ocasión se les ve más resueltas en la parte procedimental, han tomado nota de las aportaciones que se les hicieron en el caso anterior y las introducen en estos. 
Les había pedido a las alumnas que trajeran sus cartillas de vacunación. El $70 \%$ de los alumnos las trae. En la pantalla dejo una imagen del calendario vacunal infantil (CVI) de 2019 y alrededor de la mesa comenzamos a analizar las cartillas de vacunación. Empezamos por la vacuna de la Hepatitis B. Se les ve ilusionadas par estar manejando sus propias cartillas y tienen interés en saber que vacunas tienen puestas. Comparamos sus CVI con el actual, aprecian las diferencias entre el número de vacunas, que ha aumentado en estos años. Les llama la atención el cambio en cuanto al momento en que se ponen y como esto ha cambiado también. Hablamos del CVI como algo dinámico y cambiante en función del momento epidemiológico y la aparición de nuevas vacunas. Hacen preguntas concretas sobre la vacunación del Tétanos y que ocurre una vez que cumples los 14 años. Hablamos del protocolo de vacunación antitetánica. Una alumna detecta que le falta una dosis de una determinada vacuna, se interesa por saber que puede hacer y se asombra de que a sus padres "se le pasara". Aprovecho esta situación para abordar este asunto, que es más frecuente de lo esperado, y resaltar la importancia de revisar el CVI ante cualquier contacto con el menor. Les resulta muy curioso también apreciar los distintos formatos de CVI. Las alumnas que no las han traído se interesan por las de sus compañeros.

Creo que ha sido una actividad muy gratificante. El hecho de manejar sus propias cartillas ha suscitado interés por conocer que vacunas se ponen, cuando y la importancia de tener todas las dosis puestas. Es un recurso que volveré a utilizar.

Hago una síntesis de las ideas principales de la sesión y les lanzo la siguiente pregunta. ¿Cómo actuaríais ante una reacción anafiláctica en un niño que acabáis de vacunar? Se dan cuenta que han dejado un aspecto muy importante sin abordar. Dan algunas respuestas vagas. Dado que la sesión está a punto de terminar y este es un aspecto fundamental de la unidad, les pido que trabajen la respuesta en casa -actuación concreta de la enfermera 
ante una reacción anafiláctica tras vacunar-, para resolverla en la siguiente sesión.

La sesión ha transcurrido de manera dinámica y entretenida. Por mi parte me voy muy satisfecha.

\section{Tercer día}

Empezamos la sesión resolviendo la actuación de la enfermera ante una reacción anafiláctica. Se aclaran todas las dudas sobre actuación. Se les ve interesadas en concreto por la preparación y dosificación de la adrenalina. Ya que este aspecto requiere especial atención, trabajamos la preparación de la misma, realizando el procedimiento, prestando especial atención a la dilución, dosificación y vía.

Una vez dada por zanjada la unidad de vacunación, realizo una síntesis de la sesión anterior e introduzco el siguiente tema, medidas de cribado y tratamientos profilácticos en el recién nacido.

Realizo una presentación de la teoría en referencia a las medidas preventivas y de cribado recogidas en la cartilla de salud infantil mediante una presentación de power point. Es un aspecto de la salud infantil que todos desconocian en el cuestionario inicial, con lo cual pongo especial énfasis en la presentación.

Al tratar las endocrino metabolopatías, proyecto unos vídeos elaborados para profesionales del Servicio Andaluz de Salud. Es una secuencia de videos en las que se expone de manera clara y precisa todo el proceso: el programa de cribado neonatal ampliado en Andalucía, las enfermedades cribadas, la información a las familias y la prueba del talón. Les pido que tomen nota de los aspectos más relevantes y en concreto sobre el video de la prueba del talón, donde se detalla el procedimiento a realizar por la enfermera. Compruebo que les gusta el video y todas toman notas. Esto concuerda con la encuesta de satisfacción de los alumnos, donde han manifestado cuando se les preguntaba qué añadirían a los seminarios "más videos". 
Al terminar hacemos un trabajo de puesta en común de las ideas de los estudiantes y de la actuación de la enfermera.

\section{Cuarto día}

En esta sesión repetimos el ciclo metodológico con dos casos clínicos. En concreto uno relacionado con la prueba de cribado de endocrino metabolopatías y la otra con cribado auditivo, tratamiento profiláctico oftálmico y de la enfermedad hemorrágica del recién nacido. Procedemos de igual manera a las sesiones anteriores.

Veo a las alumnas involucradas en los casos clínicos. El conocer las dinámicas de trabajo les hace trabajar de una manera más eficiente. Resuelven los casos con confianza y destreza. En la parte de rol play observo una gran diferencia en relación a cuando resolvieron el primer caso clínico. Se encuentran más cómodas y agiles en la parte procedimental.

Al terminar la sesión concluyo y recapitulo sobre las ideas principales. Vuelvo a pasar el cuestionario inicial, esta vez sí les pido que se identifiquen.

Termino el ciclo con una sensación muy positiva de haber puesto en marcha todo lo propuesto e improvisar cuando lo he considerado necesario.

\section{Evaluación del aprendizaje de los alumnos}

Al finalizar el CM se realiza una valoración de nuevo de los esquemas mentales de los estudiantes y se comparan con los iniciales. Se utiliza la misma herramienta, el cuestionario inicial, y se realiza un análisis de las respuestas para conocer el grado de evolución de la clase.

Se diseñan "escaleras de aprendizaje" (Porlan, 2017) para cada pregunta, donde se aprecian los niveles iniciales y finales. Se detectan de esta manera los obstáculos superados por la mayoría de los alumnos y los que tienen margen de mejora. 
Al haber realizado el cuestionario inicial de manera anónima no he podido conocer la evolución particular de cada estudiante. Es algo que modificaré en adelante, ya que creo muy interesante conocer también este aspecto. Se presentan en las figuras 4 y 5 las escaleras de aprendizaje para dos conceptos tratados.

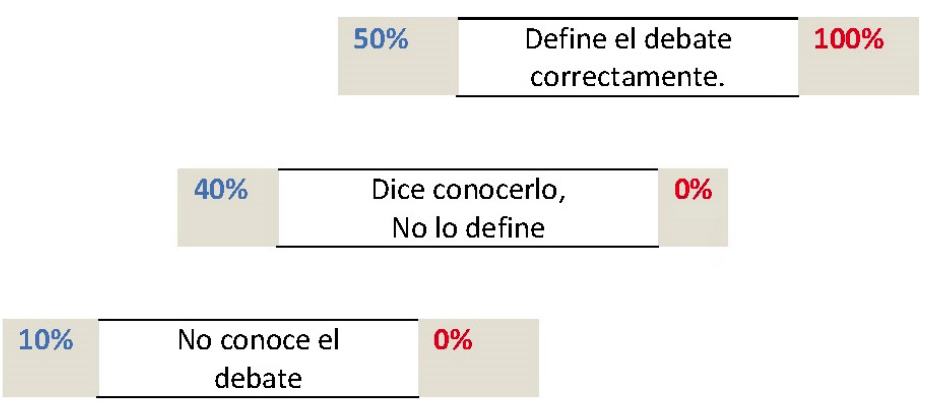

Figura 4. ¿Conoce el debate sobre vacunación Infantil?

En la escalera se puede apreciar la evolución de los alumnos en cuanto a conocimiento del debate sobre vacunación infantil. Vacunar versus no vacunar. Tras el ciclo CM el $100 \%$ de los alumnos define el debate sobre la vacunación correctamente.

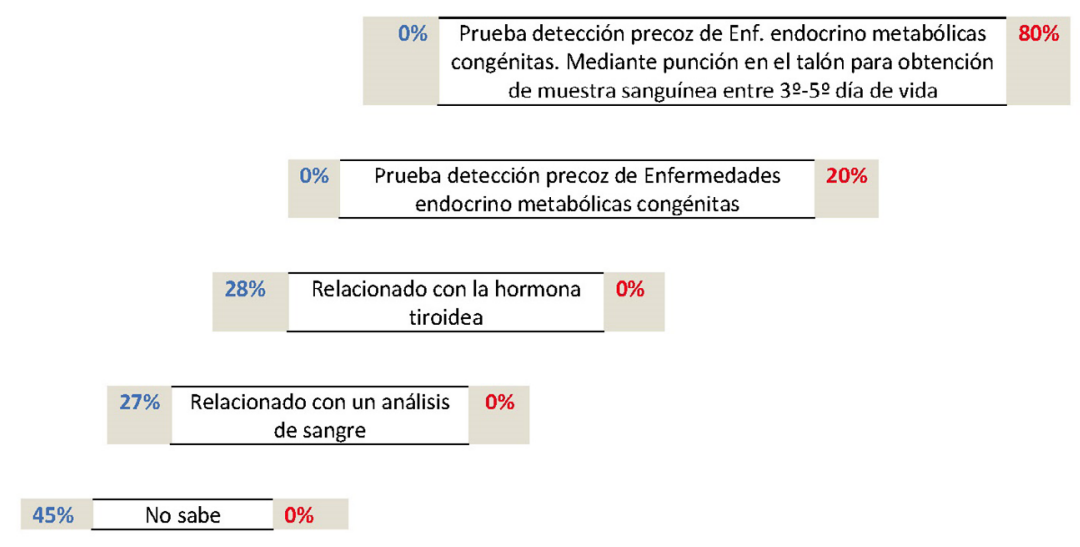

Figura 5. ¿Qué es la prueba del talón?

Jornadas de Formación e Innovación Docente del Profesorado | № 2 (2019) Esta obra se distribuye con la licencia Creative Commons Reconocimiento-NoComercial-SinObraDerivada 4.0 Internacional (CC BY-NC-ND 4.0.) 
En referencia a la prueba del talón he de decir que es donde mayor salto de aprendizaje he apreciado ya que ninguna alumna supo definirla en el cuestionario inicial, sin embargo en el final el $80 \%$ de las alumnas daba una definición completa, seguido del $20 \%$ con una respuesta correcta pero incompleta. Es decir todos los alumnos han pasado a los escalones más altos de aprendizaje.

En general ha habido un cambio considerable en la adquisición de los conceptos tratados en el CM.

Realizo también una evaluación final sobre la unidad de vacunas volviendo a utilizar el mismo cuestionario de Kahoot. Los resultados han sido los siguientes:

- Porcentaje de respuestas correctas antes del CM $61,82 \%$

- Porcentaje tras el CM fue del 96,36\%

En los resultados se aprecia que ha habido una mejora significativa en los conocimientos de los alumnos. El 80\% de los alumnos consideró la actividad divertida.

\section{Evaluación del ciclo de mejora}

\section{Satisfacción de los alumnos- evaluación docente}

Tras finalizar el CM, a los tres días, se pasa un cuestionario de google a los alumnos para evaluar la satisfacción con los seminarios del CM y su opinión sobre la docencia. Estas son las preguntas y los resultados del mismo:

¿Cómo de útil consideras estos seminarios para tu vida profesional? Escala liker.

- $90 \%$ muy útil

- 10\% útil 
¿Qué es lo que más te ha gustado de los seminarios? Respuestas cortas. Se ordenan las respuestas por frecuencia.

- Aprender y Comprender el calendario de vacunación y su importancia.

- Manejar nuestras cartillas de vacunación.

- Desmitificar los riesgos de las vacunas mediante el vídeo

- Simular la vacunación

- Reforzar los conocimientos mediante kahoot

¿Qué quitarías del seminario? Respuestas cortas.

- Nada, está bien como está 37\%

- Tener que ver el video en casa. 63\%

- ¿Qué añadirías al seminario? Respuestas cortas

- Más prácticas 63\%

- Nada, está bien como está 24.5\%

- Más videos. $12,5 \%$

Nivel de satisfacción con los seminarios. Escala liker.

- Muy satisfecha $40 \%$

- Satisfecho 60\%

- Nivel de satisfacción con la docente. Escala liker.

- Muy satisfecha $50 \%$

- Satisfecho 50\%

Teniendo en cuenta los resultados expuestos y mis propias reflexiones, quiero destacar los siguientes aspectos en la evaluación del CIMA:

Realizar una exploración de las ideas de los alumnos ha sido muy útil. Me ha servido para detectar que realmente en algunos aspectos las alumnas partían de una base mucha más baja a la esperada. Por otra parte he sido capaz de detectar algunas confusiones y conceptos erróneos. He podido reconducir o enfocar mi docencia a aclarar estos conceptos.

Jornadas de Formación e Innovación Docente del Profesorado I № 2 (2019) Esta obra se distribuye con la licencia Creative Commons 
El uso de los vídeos y el trabajo posterior sobre sus contenidos con las alumnas también ha sido una decisión acertada. Ha sido una herramienta útil para trabajar contenidos conceptuales, procedimentales y actitudinales. Las alumnas están satisfechos con su uso y han transmitido su deseo de incorporar más videos a los seminarios. El éxito del uso de videos como herramienta de apoyo al aprendizaje reside en la calidad del mismo y como aborda y presenta los conceptos a tratar. Es una labor importante del docente encontrar dichos recursos y el modo de trabar sus contenidos. En concreto para el CM, estos materiales eran muy apropiados y de calidad.

Incorporar sus CVI al seminario ha sido todo un éxito. Han mostrado interés por analizarla, conocer su estado vacunal y ha ayudado a entender el concepto de vacunación como algo dinámico y cambiante en el tiempo. Les ha acercado la teoría a la práctica y a su propia realidad. Las alumnas lo han valorado como muy positivo.

Resolver los casos clínicos e introducir la parte procedimental en los mismos es algo que también ha dado muy buen resultado. Las alumnas han manejado el material necesario y han sido capaces de simular la actuación de la enfermera. Este aspecto procedimental les motiva mucho. De hecho han calificado los seminarios muy útiles para su práctica profesional, a la vez que demandan trabajar más estos aspectos. Habrá que reflexionar sobre como aumentar el contenido procedimental.

Las alumnas han manifestado que el uso de kahoot les ha ayudado a reforzar los conocimientos, además de servir como evaluación de la adquisición de conceptos para el docente. Es algo que incorporaré a mi práctica docente.

Me ha gustado improvisar cuando lo he visto necesario. Esperaba que en la resolución de los casos se abordase la actuación ante una reacción anafiláctica. Al no ser 
así introduje la actividad para resolver en casa. Creo además que las alumnas han sentido la importancia de conocer y resolver su actuación ante esta situación de urgencia. creo que hay que estar abierto a estos imprevistos que pueden surgir en el aula, e intentar resolverlos.

Explorar las ideas de las alumnas al final de CM y analizar y comparar con los resultados iniciales ha sido muy positivo. Las escaleras de aprendizaje me han parecido una herramienta fantástica para saber lo que ha acontecido, los conceptos adquiridos y los obstáculos de aprendizaje. Sobre los que habrá que reflexionar e introducir mejoras en la docencia para superarlos. Monitorizar el proceso es fundamental.

En relación a la evaluación de las alumnas me resulta curioso que hayan hecho referencia la mayoría a la unidad de vacunación y poco a la de Salud Infantil. Esto puede ser debido a que los contenidos actitudinales se han trabajado mejor en la primera unidad. En futuras ediciones trabajaré mejor el contenido "sensibilizar en la importancia del cribado y tratamientos profilácticos neonatales".

Las alumnas están satisfechas con los seminarios, aunque existe margen de mejora en este aspecto. El nivel de satisfacción con la docente es bastante bueno. La próxima vez me gustaría analizar de una manera más exhaustiva este aspecto.

El ciclo CM me ha enseñado a participar de una manera distinta, "con la boca cerrada" como propone (Finkel, 2008). Me ha costado, pero he cedido ese "espacio" a los alumnos.

Realizar el CM ha sido una actividad muy gratificante. He trabajado mucho y en ocasiones con mucha presión por todo lo demás que sigue transcurriendo y también necesita atención. Ha merecido la pena. Me ha ofrecido 
posicionarme y ver mi docencia desde otro punto de vista. Las actividades que he planteado y realizado me han hecho sentirme muy satisfecha y han tenido muy buena valoración por parte de las alumnas. Habrá que seguir trabajando en la mejora docente diaria. Ahora me siento motivada y con las herramientas metodológicas para hacerlo.

\section{Principios didácticos}

Los principios didácticos que han guiado mi práctica docente en el CM y que pretendo mantener en mi práctica habitual son los siguientes:

- Autonomía y responsabilidad. El protagonismo del aprendizaje recae sobre el alumno, el profesor ejerce de guía y ayuda (Guerra-Marin y cols, 2017).

- Trabajo colaborativo. El grupo de alumnos interviene aportando sus ideas y conocimientos con idea de lograr una meta común (Barkley, 2007). En el caso concreto del CM, la resolución de los casos clínicos, promoviendo la integración y equilibrio entre aprendizaje práctico y teórico (Touron y Santiago, 2015).

- Aprendizaje por investigación. Los retos a los que enfrentamos a los alumnos los llevan a relacionar de forma novedosa ideas y experiencias que ya poseen (Porlan, 2017). 


\section{Referencias Bibliográficas}

Bain, K. (2005). Lo que hacen los mejores profesores universitarios. Valencia: PUV.

Barkley, E. F., Cross, K. P., \& Major, C. H. (2007). Técnicas de aprendizaje colaborativo: manual para el profesorado universitario. Madrid: Ministerio de Educación y Ciencia : Ediciones Morata.

Finkel, D. (2008). Dar clase con la boca cerrada. Valencia: Universitat de València, Servei de Publicacions.

Porlán, R. (2017). Enseñanza Universitaria, Cómo mejorarla. Madrid: Editorial Morata

Tourón, J., Santiago, R. (2015) El modelo Flipped Learning y el desarrollo del talento en la escuela: Flilpped Learning model and the development of talent at school. Ministerio de Educación. 